\title{
ASSESSMENT OF DERMATOLOGICAL LIFE QUALITY INDEX AND CARDIFF ACNE DISABILITY INDEX IN PATIENTS ATTENDING OUR COLLEGE OPD
}

\section{Dermatology}

\section{Dr. Arshi Farista*}

PG Resident, Department of Dermatology and Venerology and Leprosy, JJM Medical College, Davangere. * Corresponding Author

\section{Dr. Sowmya Manangi}

M.D. Dermatology, Ass Prof at Department of Dermatology Leprosy, JJM Medical College, Davangere.

and Venerology and

\section{ABSTRACT}

Background: Acne vulgaris is known to impair many aspects of quality of life. Acne is a common problem in adolescent children and adults

Aims: A study was undertaken to measure the impairment of quality of life of patients of acne vulgaris and correlate it with the severity of lesions. This study was aimed to assess the impact of acne and its sequelae on the DQLI and CADI

Materials and methods: This was a hospital based prospective study done between September 2018 to march 2019 on 170 patients coming to our college opd. Acne vulgaris and its sequelae were graded using global acne grading system. The DLQI and CADI score were recorded by giving the patients a set of questionnaire. General use of cosmetics and other history related to diet and habits were noted.

Results: the over all CADI SCORE were low indicating a mild impairement. Out of 170 patients 110 were girls and 60 were boys. Almost $95 \%$ patients had applied fair and lovely. The severity of acne progressively lessened in older patients. Mean DLQI score was 5.3. DQLI score were statistically influenced by the age of the patient, duration of andgrade of acne, acne scars and post hyperpigmentation.

Conclusion: the study showed CADI SCORE AND DLQI were low indicating mild imapirement. Assurance and counseling along with early treatment of acne vulgaris are important to reduce disease-related psychosocial sequelae and increase the efficacy of treatment.

\section{KEYWORDS}

\section{DQLI, CADI, INDEX, ACNE VULGARIS}

\section{INTRODUCTION}

Acne vulgaris is a chronic inflammatory disease of the pilosebaceous unit characterized by seborrhea, open and closed comedones, papules, pustules, and in more severe cases, nodules and pseudocysts. It commonly affects the face, upper chest, and upper back. More than $85 \%$ of adolescents suffer from acne and in $50 \%$ cases, it extends into adulthood. The major complications of acne are scarring and psychosocial distress which persists long after active lesions have disappeared. Psychosocial effects of acne vulgaris have been long identified, but this sequelae of acne remain under evaluated.

Patients with acne have been shown to have levels of social, psychological, and emotional impairments similar to serious diseases such as asthma, epilepsy, diabetes, or arthritis. These patients are more prone to embarrassment, social withdrawal, depression, anxiety, and anger.

Evaluation of acne using only clinical assessment does not capture the impact of the disease adequately. Assessment of impact on healthrelated quality of life $(\mathrm{QoL})$ is needed to fully characterize the overall disease burden and effectiveness of treatment. The use of QoL questionnaires can help us adequately understand how acne affects the patient on a day-to-day basis and can aid in assessing the efficacy of therapy and design more targeted interventions.

\section{Materials and Methods}

It was a hospital-based, prospective, cross-sectional study done in our college OPD between September 2018 to march 2019 (6 months) on 170 patients. Patients aged 15 years and above with a clinical diagnosis of acne vulgaris were included in the study . Patients who used topical and systemic drugs known to predispose them to acne, and nonconsenting patients were excluded.

A detailed history pertaining to socio-demographic data, presenting complaints, duration of acne, etc., were elicited. Cutaneous examination was done on all patients and the following were noted:

(i) Type of skin (dry/normal/oily),

(ii) site of lesion (face, chest, or back),

(iii) grade of acne,

(iv) postacne hyperpigmentation (present/absent),

(v) acne scars.
Grade II: Papules, comedones, few pustules

Grade III: Predominant pustules, nodules, abscesses

Grade IV: Mainly cysts, abscesses, widespread scarring.

All patients were asked to respond to 2 questionnaire (scales) CADI and DLQI. The DLQI questionnaire, first introduced by Finlay and Khan, in 1994 was used as the study instrument for this study.

DLQI is a validated questionnaire which grades QoL by assessing the following domains: (a) physical symptoms and feelings (questions 1 and 2), (b) daily activities (questions 3 and 4), (c) leisure (questions 5 and 6), (d) work/school (questions 7), (e) personal relationships (questions 8 and 9), and (f) treatment (question 10). Each question is scored as "very much" (score 3), "a lot" (score 2), "a little" (score 1), and "not at all" (score 0), keeping in mind the problems faced the previous week due to the disease.

Final DLQI score is the sum of all scores (range 0-30). High scores indicate poor QoL. Patients were asked to fill up the DLQI questionnaire

DLQI score interpretation is done as follows:

$0-1$ no effect on patient's life

$2-5$ small effect on patient's life

6-10 moderate effect on patient's life

$11-20$ very large effect on patient's life

21-30 extremely large effect on patient's life.

CADI is a questionnaire which is specific for acne and contains 5 questions-related to the last month- about feelings, interference with social life and interaction with the opposite gender, avoidance of public places, appearance of the skin and perceived severity of disease state. Each question is scored from 0-3 leading to a total score of 0-15. A higher score shows a very large impact on quality of life. A CADI score $<8$ is considered to have a small effect on quality of life and a score $>8$ is considered as having a larger effect on QOL.

\section{Statistical analysis}

Data was entered into SPSS version 21.0 and represented in proportions and percentages. Data was analyzed to compare the quality of life indices (CADI \& DLQI) for duration and severity of acne.

Result

A total of 170 patients aged between 15 to 30 years were taken in the 
study. Out of 170 patients, 110 were females and 60 were males. The severity of acne progressively lessened in older patients. $18-25$ years age group was mainly affected, followed by $16-17$ years. Mean age of study was 20 years. There was a female preponderance. $80 \%$ patients were unmarried.

In the present study, $60 \%$ of patient presented with lesions over the face followed by lesions of face and back followed by lesions over face, back and chest. $30 \%$ of patients showed post inflammatory hyperpigmentation and $13 \%$ had scarring.

Quality of life measures in acne vulgaris patients

\begin{tabular}{|l|c|c|}
\hline Dermatology life quality index (DLQI) grade & & \\
\hline & $\mathrm{n}=$ & Percentage \\
\hline No effect (0-1) & 7 & 4.3 \\
\hline Small effect (2-5) & 44 & 26.3 \\
\hline Moderate effect (6-10) & 68 & 38.7 \\
\hline Very large effect (11-20) & 49 & 29 \\
\hline Extremely large effect (21-30) & 2 & 1.7 \\
\hline Cardiff acne disability index (CADI) grades & & \\
\hline Low (0-4) & 53 & 31 \\
\hline Medium (5-9) & 97 & 56 \\
\hline High (10-14) & 20 & 12 \\
\hline
\end{tabular}

According to DLQI scores of acne showed no effect in $4.3 \%$ of the patients, small effect in $26.3 \%$ of the patients, moderate effect in $38.7 \%$ of patients, very large effect in $29 \%$ of patients and extremely large effects on $1.7 \%$ of patients.

According to CADI scores of acne showed low effect in $31.3 \%$ of the patients, majority had medium effect in $56.7 \%$ of patients and high effect was seen on $12 \%$ of patients.

Mean (SD) Scores of DLQI and CADI were 8.61 (4.99) and 6.22 (2.73) respectively

\section{CONCLUSION}

The Present study showed CADI score were low indicating mild impairment and showed that quality of life is significantly impaired in patients of severe acne vulgaris. The impact of acne on QOL can be measured using general health measures, dermatology specific measures and acne specific measures. The study demonstrated that CADI and DLQI were easily understood by general population and quickly completed.

This study thus stresses the importance of assurance and counseling along with early treatment of acne vulgaris in reducing disease-related psychosocial sequelae and enhancing the efficacy of treatment.

\section{REFERENCES}

1) Hazarika N, Rajaprabha RK. Assessment of life quality index among patients with acne vulgaris in a suburban population. Indian J Dermatol 2016;61:163-8

2) Finlay AY. Quality of life indices. Indian J Dermatol Venereol Leprol 2004;70:143-8

3) Raju BP, Nagaraju U. Quality of life among adolescents with acne in a tertiary referral centre in Bangalore, South India. Indian J Paediatr Dermatol 2017;18:94-9

4) Durai PT, Nair DG. Acne vulgaris and quality of life among young adults in South India. Indian J Dermatol 2015;60:33-40

5) Gupta A, Sharma YK, Dash KN, Chaudhari ND, Jethani S. Quality of life in acne vulgaris: Relationship to clinical severity and demographic data. Indian J Dermatol Venereol Leprol 2016;82:292-7 